\title{
Decorrelation of edge plasma turbulence at the transition from low- to high-confinement mode in the DIII-D Tokamak
}

\author{
S. Coda ${ }^{\text {a, } *, 1}$, M. Porkolab ${ }^{\text {a }}$, K.H. Burrell ${ }^{\mathrm{b}}$ \\ ${ }^{\text {a }}$ Department of Physics and Plasma Science and Fusion Center, Massachusetts Institute of Technology, Cambridge, MA 02139, USA \\ ${ }^{\mathrm{b}}$ General Atomics, P.O. Box 85608, San Diego, CA, 92186-5608, USA \\ Received 16 June 2000; accepted 19 June 2000 \\ Communicated by V.M. Agranovich
}

\begin{abstract}
The modification of turbulence in the edge plasma of the DIII-D tokamak at the transition from the low to the high mode of confinement is investigated with a phase-contrast imaging diagnostic. The amplitude and radial correlation length of the turbulence in the confinement region decrease at the transition, whereas the decorrelation time increases. The transition model of Biglari, Diamond, and Terry [Phys. Fluids B 2 (1990) 1], based on turbulence decorrelation by $\boldsymbol{E} \times \boldsymbol{B}$ velocity shear, is quantitatively substantiated by measurements of the theoretical control parameter. Further quantitative predictions of the theory are tested for the first time. (C) 2000 Published by Elsevier Science B.V.
\end{abstract}

\section{Introduction}

A central goal of research in nuclear fusion by magnetic confinement has been the minimization of anomalous plasma transport, defined as all transport in excess of the inescapable rate due to Coulomb collisions. Indeed, a great deal of progress has been

\footnotetext{
* Corresponding author. Tel.: 4121693 3465; fax: 4121693 5176.

E-mail address: stefano.coda@epfl.ch (S. Coda).

${ }^{1}$ Present and permanent address: Centre de Recherches en Physique des Plasmas, Ecole Polytechnique Fédérale de Lausanne, CH-1015 Lausanne, Switzerland.
}

made over the past few decades, which have seen the successive discoveries of various regimes of improved confinement [1]. Much current research aims at improving the understanding and control of these regimes. Anomalous transport is widely attributed to turbulent fluctuations caused by microinstabilities $[1,2]$. However, the experimental evidence for this connection is still incomplete, and much remains unknown about the nature and properties of the turbulence and of the underlying instabilities. More generally, understanding turbulence, in both neutral and charged fluids, is widely perceived as one of the remaining great scientific challenges of classical physics [3].

The first and most universal of the enhanced confinement regimes is the high-confinement mode 
(H-mode) [4]. There is now considerable evidence that a single mechanism, turbulence decorrelation and suppression by a sheared $\boldsymbol{E} \times \boldsymbol{B}$ velocity, is responsible for the transition from the low-confinement mode (L-mode) to the H-mode [5]. This same mechanism is thought to play an important role in the enhanced-confinement regimes discovered more recently [6]. A theoretical model for the shear decorrelation mechanism was proposed nearly a decade ago [7] and by now has become widely accepted, with additional support from recent numerical simulations [8]. This model, which implies a self-organization of the plasma into a higher-energy yet less turbulent state, departs radically from conventional wisdom and constitutes a fundamental new addition to the understanding of turbulence. Measurements by electrostatic probes [9] and reflectometry [10] have provided initial corroboration of the theory.

An experimental test of the predictions of the model requires the simultaneous measurement with good spatial and temporal resolution of the correlation length and decorrelation time of the turbulence, as well as of the electric field, in the plasma edge. In addition, the decorrelation time should be measured in the plasma frame of reference, to avoid distortions caused by Doppler broadening from the sheared plasma flow. In this Letter, we present the first simultaneous, time-resolved, noninvasive measurement of these correlation parameters in the plasma frame across the $\mathrm{L}-\mathrm{H}$ transition. The measurement was performed with a high-spatial-resolution phasecontrast imaging (PCI) diagnostic [11] in the DIII-D tokamak [12]. Used in conjunction with measurements of the electric field by charge-exchange recombination (CER) spectroscopy [13], the PCI data permit a quantitative test of the theoretical criterion for shear decorrelation, which is found to be well satisfied. In addition, we find that the radial correlation length, as well as the amplitude, of the turbulent density fluctuations in the confinement region decrease at the transition, whereas the decorrelation time increases. These measurements make possible for the first time a truly complete test of the theory, as they allow us to test detailed quantitative predictions generated by two versions of the model $[7,14]$ for the changes in those correlation quantities. Incomplete agreement is obtained in both cases, suggesting that refinements of the theory are desirable in order to achieve a more complete understanding of the $\mathrm{L}-\mathrm{H}$ transition.

The inception of the H-mode, related to the attainment of a heating power threshold, is generally abrupt and is characterized by the formation of a transport barrier in the plasma edge, concomitant with a reduction in the edge turbulence level. The $\mathrm{L}-\mathrm{H}$ transition is empirically identified by a decrease in the spectroscopic $\mathrm{D}_{\alpha}$ edge emission, which indicates reduced edge particle transport. On a slower time scale, the confinement improvement extends to the core $[1,15]$. During the evolution of the H-mode, Edge Localized Modes (ELMs) - quasiperiodic edge instabilities - are often observed. In the remainder of this Letter we shall only consider the early, ELM-free $\mathrm{H}$-mode phase occupying the first few tens of milliseconds after the $\mathrm{L}-\mathrm{H}$ transition. The properties of turbulence change substantially in the later phase, especially in the presence of ELMs.

\section{Experimental setup}

The PCI diagnostic [16] employs a 20-W $\mathrm{CO}_{2}$ laser beam of $10.6 \mu \mathrm{m}$ wavelength and $7.6 \mathrm{~cm}$ width, which is launched vertically through the outer plasma edge of DIII-D (see Fig. 1) and is then passed through a Zernike phase-contrast filter [17] to create a radial image of the plasma onto a 16-element HgMnTe linear detector array. Thus, the system measures the vertical line integrals of the density fluctuations at 16 radial locations, typically distributed from just inside to just outside the last closed flux surface (LCFS). Any eight of the 16 signals can be collected in a given plasma discharge. The radial resolution is $0.5 \mathrm{~cm}$ and the temporal resolution is $0.5 \mu \mathrm{s}$; the system is sensitive to density fluctuations as low as $10^{15} \mathrm{~m}^{-3}$, and the signalto-noise ratio is typically in the range 10-100. These properties ensure good statistics for correlation analysis. The diagnostic is absolutely calibrated by means of acoustic waves in air. While the accuracy on the absolute calibration is $\sim 30-40 \%$, the relative amplitude from chord to chord is known to an accuracy of $\sim 10-15 \%$.

The frequency range is $8-1000 \mathrm{kHz}$, the lower limit being imposed by mechanical vibrations; the wave-number range is $0.65-6 \mathrm{~cm}^{-1}$. The vertical 


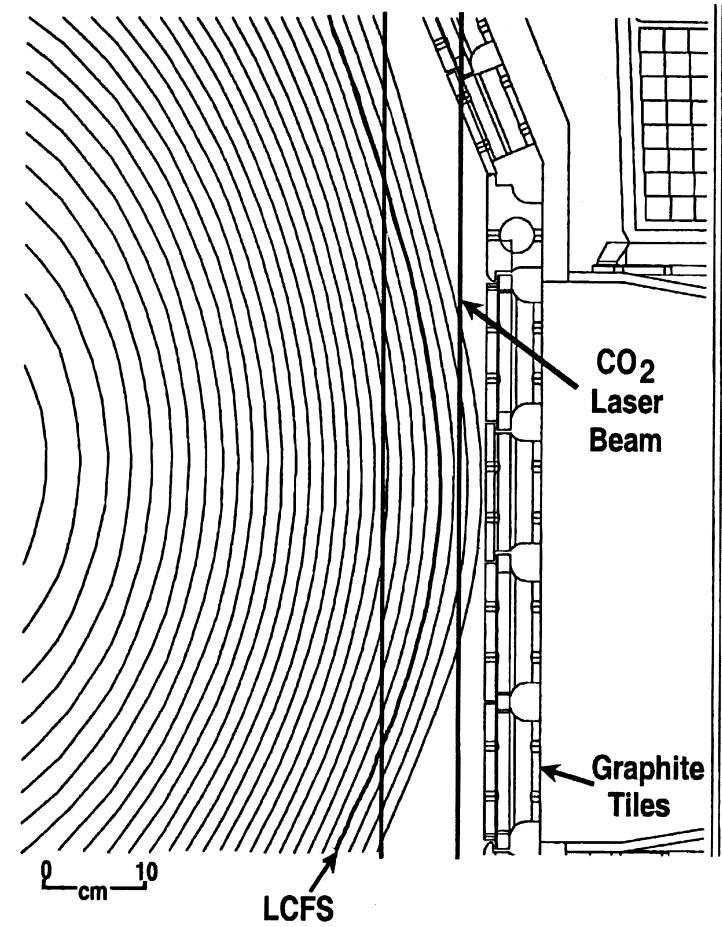

Fig. 1. Geometry of the PCI measurement. The outer portions of the magnetic flux surfaces of a typical DIII-D plasma are shown. The position of the last closed flux surface (LCFS) can vary from discharge to discharge.

integration combined with the field-line geometry at the edge constrains the measured wave vectors to be essentially radial, i.e., perpendicular to the magnetic flux surfaces. As a result, the measured frequency spectra experience negligible Doppler shifts in response to the poloidal and toroidal $\boldsymbol{E} \times \boldsymbol{B}$ drifts due to the radial electric field; in addition, the measured correlation length can be identified with the radial correlation length. These conclusions were confirmed by extensive analytical and numerical modeling $[16,18]$. We emphasize that only turbulent fluctuations are considered in the present work; since the nature of the data is profoundly altered by the presence of coherent fluctuations, such cases were excluded from this study.

Experimental tests of theory require the estimation of the correlation length and decorrelation time of turbulent plasma eddies [7]. Since an eddy travels at the group velocity of the fluctuations, a Lagrangian rather than a Eulerian approach is required to determine its true lifetime. To this effect we calculate the autocorrelation function of the peak spatial Fourier component of the turbulence. The intrinsic eddy decorrelation time is taken to be the $e$-folding time of this function [16] and is systematically larger than the stationary (Eulerian) decorrelation time, as expected. The applicability of this approach, which can intuitively be seen as a measurement in the eddy frame of reference, is contingent on the lack of Doppler broadening. The radial correlation length is estimated as the $e$-folding length of the envelope of the equal-time spatial correlation coefficient, fitted by a Gaussian.

\section{Phenomenology of edge turbulence in L- and H-mode in DIII-D}

The properties of turbulence in L- and H-mode have been studied with the PCI system in detail in over 100 DIII-D diverted deuterium discharges, in the region from $6 \mathrm{~cm}$ inside to $4 \mathrm{~cm}$ outside the last closed flux surface. Several quantitative features exhibit significant dependences on plasma parameters, which are beyond the scope of this Letter [16]. Here we shall focus on the systematic changes occurring at the $\mathrm{L}-\mathrm{H}$ transition. For simplicity, we shall identify each PCI chord by its intersection with the midplane; however, it must be stressed that each signal contains contributions from the ensemble of flux surfaces with minor radii equal or larger than the minor radius at the midplane.

Inside the LCFS, the signal amplitude is lower in $\mathrm{H}$-mode than in L-mode [19]. The reduction factor is largest in the region just inside the LCFS and ranges from 1.2 to 3 in our data set; further into the plasma, 4-6 cm inside the LCFS, that factor is generally close to 1 . In the region of open field lines (scrape-off layer, or SOL), the H-mode amplitude can be slightly smaller than its L-mode counterpart, but it is also often larger by up to a factor of two. Representative radial profiles of the density fluctuations, obtained by using a set of similar discharges with different positions of the LCFS, are shown in Fig. 2. The peak located $0.5-1.5 \mathrm{~cm}$ inside the LCFS is a universal feature, although it is systematically less prominent in $\mathrm{H}$-mode. It must be remembered that these data represent the rms values of the line-integrated den- 


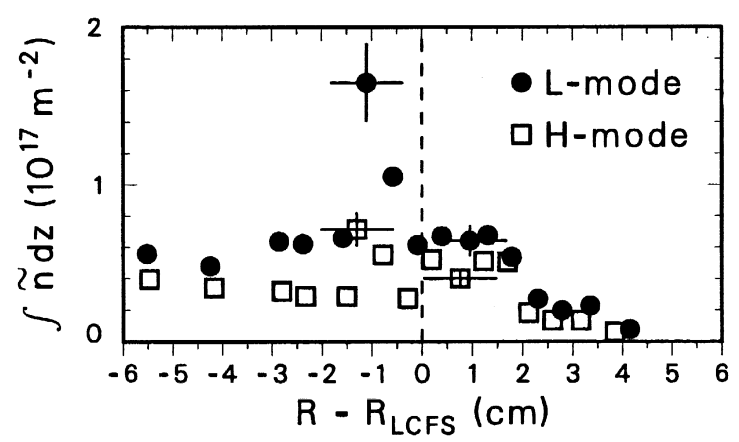

Fig. 2. Spatial distribution of rms values of line integrals of density fluctuations in L- and $\mathrm{H}$-mode, as a function of horizontal distance from LCFS on the midplane. Data are collected from a set of similar shots with varying LCFS position (magnetic field $B_{\phi}=2.1 \mathrm{~T}$, current $I_{p}=1.35 \mathrm{MA}$, line-averaged density $\bar{n}_{e}=4 \times$ $10^{19} \mathrm{~m}^{-3}$, input neutral-beam power $1.1-2.3 \mathrm{MW}$ ). The error bars shown reflect chord-to-chord calibration uncertainties; the common absolute uncertainty is $40 \%$.

sity fluctuations; thus, part of the signal reduction in $\mathrm{H}$-mode could be due to a decrease in the vertical correlation length.

The frequency spectrum is monotonically decreasing everywhere in L-mode, generally with an approximate $\omega^{-2}$ dependence. In H-mode, the spectrum is broader overall than in L-mode but exhibits irregular multiple peaks. These features are reflected in the $\mathrm{H}$-mode autocorrelation function, which is narrower at small delay times but exhibits a more prominent tail at large delays than its L-mode counterpart. The corresponding decorrelation time is generally larger in $\mathrm{H}$-mode than in L-mode in the region from the LCFS to $1.5 \mathrm{~cm}$ inside, smaller elsewhere; its value in all cases varies between 10 and $50 \mu \mathrm{s}$. The intrinsic eddy decorrelation time, while systematically larger, displays the same relative phenomenology: typical values in the region just inside the LCFS are $20-40 \mu \mathrm{s}$ in L-mode, $40-80 \mu \mathrm{s}$ in H-mode. It should be noted that the frequency components below $8 \mathrm{kHz}$, inaccessible to the measurements, could further increase these values: hence, these times should be taken as lower limits.

The radial correlation length decreases at the $\mathrm{L}-\mathrm{H}$ transition inside the LCFS, while it remains constant or increases, sometimes substantially, in the SOL. The reduction factor in the confinement region ranges from 1.3 to 3 , with values from 1.2 to $3.5 \mathrm{~cm}$ in L-mode and from 0.4 to $1.2 \mathrm{~cm}$ in H-mode, depending on plasma parameters. In the SOL, the correlation length is generally shorter than $1 \mathrm{~cm}$ in L-mode, while values up to $3 \mathrm{~cm}$ have been documented in H-mode [16].

Owing to the line integration, the measurements made on chords nominally inside the LCFS are in fact affected by the turbulence in the SOL also. However, the length of the integration path across the SOL decreases rapidly with decreasing major radius for those chords. The strong peaking of the fluctuation signal $0.5-1.5 \mathrm{~cm}$ inside the LCFS in L-mode and the strikingly different phenomenology of the correlation properties inside and outside the LCFS (in both L- and H-mode) suggest that the measurements inside the LCFS are little affected by the SOL. The contribution of a chord segment to the mean square signal is proportional to $L_{z} \mathscr{L}_{z} \tilde{n}^{2}$, where $L_{z}$ is the length of the segment, $\mathscr{L}_{z}$ is the vertical correlation length, and $\tilde{n}$ is the local density fluctuation amplitude. The contributions from the outer layers can be subtracted in principle by using the measurements on the outer chords, weighted by their effective integration lengths. A subtraction algorithm based on this principle has confirmed the qualitative conclusion that the SOL turbulence has little effect on the measurements performed on the chords inside the confinement region. However, this method is very sensitive to calibration uncertainties, to uncertainties in the radial position (the LCFS position is known to an accuracy of $\pm 0.5 \mathrm{~cm}$ ), and to strong gradients in both $\tilde{n}$ and $\mathscr{L}_{z}$. For these reasons, this subtraction algorithm, while adequate for separating the turbulence in the SOL from that inside the LCFS, has not been deemed sufficiently robust for a more quantitative analysis aimed at unfolding the distribution of the local density fluctuations.

The fluctuation suppression always appears to be simultaneous with the $\mathrm{L}-\mathrm{H}$ transition, as defined by the start of the decrease in the $\mathrm{D}_{\alpha}$ signal, and occurs in less than $0.1 \mathrm{~ms}$. The integration time for the estimation of the correlation parameters is dictated by statistical requirements, and is typically $5 \mathrm{~ms}$. Within this resolution, the change in the correlation length is also seen immediately after the transition, i.e., in the first interval lying entirely in H-mode. The changes in the decorrelation time are more 


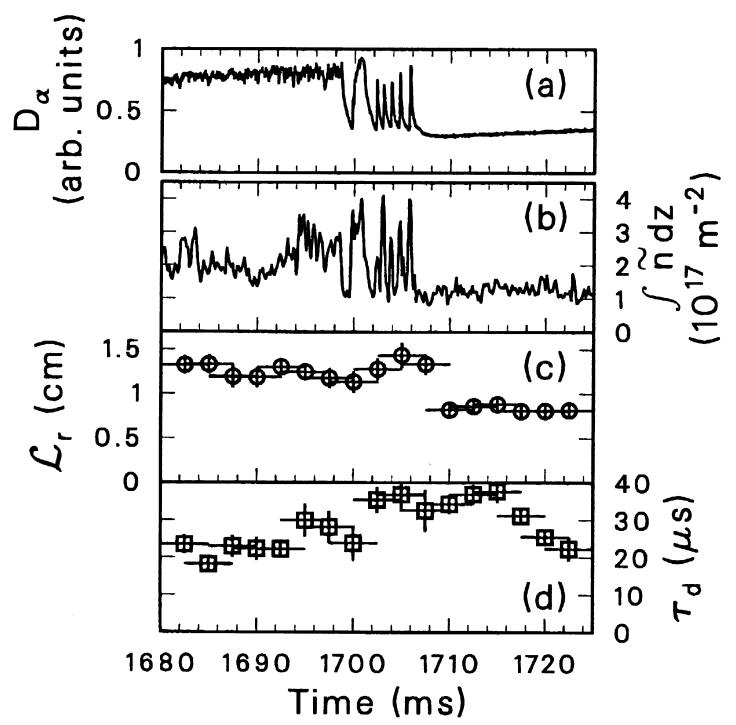

Fig. 3. Evolution across the $\mathrm{L}-\mathrm{H}$ transition of (a) $\mathrm{D}_{\alpha}$ emission, (b) rms value of line-integrated density fluctuations (40\% uncertainty on the absolute value), (c) radial correlation length, and (d) intrinsic eddy decorrelation time. Measurements (b)-(d) are made with PCI in the region from the LCFS to $0.8 \mathrm{~cm}$ inside it. Plasma parameters: $B_{\phi}=2.1 \mathrm{~T}, I_{p}=1.25 \mathrm{MA}, \bar{n}_{e}=3.5 \times 10^{19} \mathrm{~m}^{-3}$, input neutral-beam power $6.2 \mathrm{MW}$.

erratic. This is shown in Fig. 3 for the case of a dithering transition.

\section{Tests of the shear decorrelation theory}

The reduction in transport is believed to be caused by sheared poloidal $\boldsymbol{E} \times \boldsymbol{B}$ flows inducing decorrelation between differentially rotating eddies [7]. This causal relation has been corroborated by CER measurements of the edge radial electric field with $0.5-\mathrm{cm}$ resolution in DIII-D, which have shown an increase in the $\boldsymbol{E}$ shear just before the L-H transition [20]; the field forms a well structure with a minimum just inside the LCFS. The analysis of the $\boldsymbol{E} \times \boldsymbol{B}$ shear decorrelation effect, carried out initially by Biglari, Diamond, and Terry (BDT) [7] in cylindrical geometry, was later extended by the full toroidal formulation of Hahm [21], subsequently completed by Hahm and Burrell (HB) [14]. According to this analysis, the $\mathrm{H}$-mode state is characterized by the condition $\omega_{s}$ $\gg \omega_{T}$, where $\omega_{s} \equiv v_{\boldsymbol{E} \times \boldsymbol{B}}^{\prime} \mathscr{L}_{r} / \mathscr{L}_{\theta}$ is the shearing rate, $v_{\boldsymbol{E} \times \boldsymbol{B}}^{\prime}=\left(R B_{\theta} / B_{\phi}\right) \partial\left[E_{r} /\left(R B_{\theta}\right)\right] / \partial r, R$ is the major radius, $r$ is the minor radius, $B_{\theta}$ and $B_{\phi}$ are the poloidal and toroidal magnetic fields respectively, $\mathscr{L}_{r}$ and $\mathscr{L}_{\theta}$ are the radial and poloidal correlation lengths respectively, $\omega_{T} \equiv 1 / \tau_{d}$, and $\tau_{d}$ is the eddy decorrelation time; the turbulence quantities here refer to the state with no $\boldsymbol{E}$ shear (L-mode). This asymptotic criterion has been widely accepted and has appeared as a key ingredient in a number of other theories [1]. With $\mathscr{L}_{r}$ and $\tau_{d}$ provided by PCI and $v_{\boldsymbol{E} \times \boldsymbol{B}}^{\prime}$ supplied by CER, the greatest uncertainty in a test of the inequality comes from a lack of accurate measurements of $\mathscr{L}_{\theta}$; from far-infrared scattering spectra [22], we estimate $\mathscr{L}_{\theta}=3 \pm 1 \mathrm{~cm}$ [23].

Fig. 4 shows a comparison of $\omega_{s}$ and $\omega_{T}$ in Land $\mathrm{H}$-mode in the region where the transport barrier is created. Averaging from PCI line integration here is effected over the outermost $5 \%$ in poloidal flux, or approximately $2 \mathrm{~cm}$ in minor radius. Clearly the condition $\omega_{s} \gg \omega_{T}$ is well satisfied in H-mode, whereas the two quantities are comparable in L-mode, as should be expected for the phase leading up to the transition. These statements hold true for all the discharges to which this test has been applied. As was mentioned before, it is possible that $\omega_{T}$ is overestimated by the lack of access to the lowest frequencies; if this were the case, the inequality

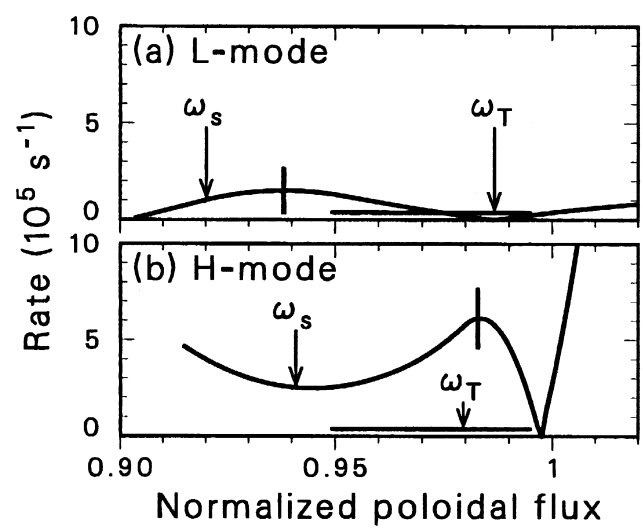

Fig. 4. Comparison of shearing rate and turbulence decorrelation rate in $\mathrm{L}$ - and $\mathrm{H}$-mode $(25 \mathrm{~ms}$ before and after the transition, respectively). The decorrelation rate is shown as a segment over the spatial range sampled by PCI to obtain its value. The abscissa is a normalized radial coordinate ( $=1$ at the LCFS). Plasma parameters: $B_{\phi}=2.16 \mathrm{~T}, I_{p}=1.54 \mathrm{MA}, \bar{n}_{e}=3 \times 10^{19} \mathrm{~m}^{-3}$, input neutral-beam power $8.5 \mathrm{MW}$. 
would be satisfied to an even greater degree. We emphasize again that these results apply to the $\mathrm{L}-\mathrm{H}$ transition; there are indications that in the fully evolved H-mode other effects, such as flow curvature and dephasing between potential and density fluctuations [24], are at least partly responsible for the continued low rate of transport.

As a result of the different formalisms employed by the BDT and HB models (cylindrical and ballooning, respectively), different predictions are obtained for the changes in the radial correlation length and in the decorrelation time from $\mathrm{L}$ - to $\mathrm{H}$-mode as functions of the $\mathrm{H}$-mode shearing rate. The BDT formulas are $\mathscr{L}_{r \mathrm{H}} / \mathscr{L}_{r \mathrm{~L}} \simeq\left(2 \omega_{s \mathrm{H}} \tau_{d \mathrm{~L}}\right)^{-1 / 3}$ and $\tau_{d \mathrm{H}} / \tau_{d \mathrm{~L}} \simeq$ $\left(2 \omega_{s \mathrm{H}} \tau_{d \mathrm{~L}}\right)^{-2 / 3}$; the HB formulas are $\mathscr{L}_{r \mathrm{H}} / \mathscr{L}_{r \mathrm{~L}} \simeq(1$ $\left.+\omega_{s \mathrm{H}}^{2} \tau_{d \mathrm{~L}}^{2}\right)^{-1 / 2}$ and $\tau_{d \mathrm{H}} / \tau_{d \mathrm{~L}} \simeq 1$ (neglecting residual logarithmic dependences). A test of these predictions on a representative set of discharges is shown in Fig. 5. It is apparent that the BDT theory provides a better fit to the spatial correlation data, whereas the HB theory is a better predictor of the temporal correlation data. These incomplete agreements may be attributed to the non-self-consistent nature of both models, which do not consider the reaction of the turbulence on the flows and, through the modification of transport, on the plasma parameters. While the evidence strongly supports the identification of $\omega_{s} / \omega_{T}$ as the control parameter for the $\mathrm{L}-\mathrm{H}$ transition, the detailed changes occurring in the turbulence spectrum can be expected to be affected by the rapid variations in plasma conditions, particularly the pressure gradient, that occur at the transition. These variations are ignored by the theories considered here. Conversely, in self-consistent theories [25] the decorrelation time has been modeled only in an ad hoc manner, with a dependence $\tau_{d} \propto \omega_{s}^{-2}$ which is even less satisfactory than the BDT prediction. Indeed, $\tau_{d}$ is experimentally found to increase at the $\mathrm{L}-\mathrm{H}$ transition. This may simply indicate an increased stability of the modes, which leads to a smaller growth rate and thus to a larger decorrelation time; or it could be argued that the chief L-mode instabilities (e.g., ion-temperature-gradient modes [1]) are drastically quenched in $\mathrm{H}$-mode and are replaced by new and less virulent instabilities [24]. In this case, a proper test of the predictions discussed above would require an isolated analysis of the modes that are suppressed, a very difficult task in practice.

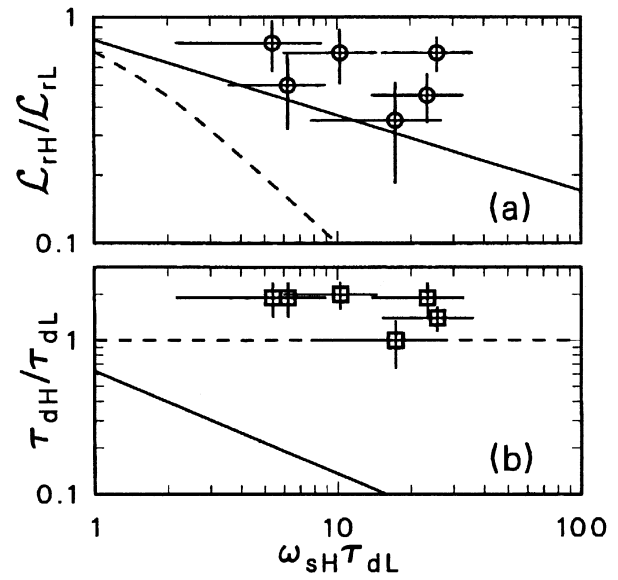

Fig. 5. Ratios of (a) radial correlation lengths and (b) decorrelation times in $\mathrm{H}$ - and L-mode, as functions of the H-mode shearing rate: experimental values averaged in the region of normalized poloidal flux 0.95-1 (open symbols), BDT predictions (solid lines), HB predictions (dashed lines).

A complete self-consistent theory of the $\mathrm{L}-\mathrm{H}$ transition would also require an explicit calculation of the anomalous diffusivity. Only heuristic estimates can be used at present. In a random-walk model, the diffusivity $D_{\text {rw }}=\mathscr{L}_{r}^{2} / \tau_{d}$ calculated from the data of Fig. 5 decreases by a factor ranging from 2.5 to 10 from $\mathrm{L}$ - to $\mathrm{H}$-mode, more than the twofold increase typically observed in the confinement time. However, it must be remembered that the PCI measurements are performed at the edge, where the transport barrier forms first, while the confinement time is a global quantity. In addition, turbulence suppression is known to be stronger at the outer than at the inner edge [10]. It can thus be argued that the local diffusivity (a quantity that is difficult to measure accurately at the edge) may decrease by more than a factor of two, as suggested by the random-walk estimate.

\section{Conclusions}

In conclusion, the first nonperturbing, simultaneous measurement of the radial correlation length and decorrelation time of the turbulence with high temporal and spatial resolution has been carried out in the edge plasma of the DIII-D tokamak. The results presented here are quantitatively consistent with the 
theoretical prediction that the $\boldsymbol{E} \times \boldsymbol{B}$ flow shearing rate should greatly exceed the intrinsic turbulence decorrelation rate in $\mathrm{H}$-mode and that the two rates should be comparable just before the L- to H-mode transition. This supports the paradigm of turbulence decorrelation by a sheared $\boldsymbol{E} \times \boldsymbol{B}$ flow producing the improved-confinement, $\mathrm{H}$-mode state $[7,14]$. However, comparison of $\mathscr{L}_{r}$ and $\tau_{d}$ before and after the $\mathrm{L}-\mathrm{H}$ transition shows that only some of the detailed quantitative predictions of the simple analytic theories $[7,14]$ are correct. This indicates the need for more complete theories which self-consistently include the effect of the drastic changes in background plasma conditions that occur after the transition.

\section{Acknowledgements}

The authors are grateful to the DIII-D team for the operation of the tokamak, heating systems, and diagnostics, and to B.A. Carreras, P.H. Diamond, and T.S. Hahm for illuminating discussions. This work was supported by the US Department of Energy under Grant No. DE-FG02-91ER54109 and Contract No. DE-AC03-89ER51114.

\section{References}

[1] See B.A. Carreras, IEEE Trans. Plasma Sci. 25 (1997) 1281, and references therein.

[2] J.D. Callen, Phys. Fluids B 4 (1992) 2142; R.J. Groebner, Phys. Fluids 5 (1993) 2343.

[3] U. Frisch, Turbulence (The Legacy of A.N. Kolmogorov), Cambridge University Press, Cambridge, UK, 1995.

[4] F. Wagner et al., Phys. Rev. Lett. 49 (1982) 1408.

[5] K.H. Burrell et al., Phys. Plasmas 1 (1994) 1536.
[6] K.H. Burrell, Science 281 (1998) 1816.

[7] H. Biglari, P.H. Diamond, P.W. Terry, Phys. Fluids B 2 (1990) 1

[8] Z. Lin et al., Science 281 (1998) 1835.

[9] Ch.P. Ritz et al., Phys. Rev. Lett. 65 (1990) 2543; G.R. Tynan et al., Phys. Plasmas 1 (1994) 3301; S. Ohdachi et al., Plasma Phys. Control. Fusion 36 (1994) A201.

[10] E.J. Doyle et al., in: Proc. 14th International Conference on Plasma Physics and Controlled Nuclear Fusion Research, Würzburg, 1992 (International Atomic Energy Agency, Vienna, Austria, 1993), vol. 1, p. 235.

[11] S. Coda, M. Porkolab, T.N. Carlstrom, Rev. Sci. Instrum. 63 (1992) 4974.

[12] J.L. Luxon, L.G. Davis, Fusion Technol. 8 (1985) 441.

[13] P. Gohil et al., in: Proc. 14th IEEE/NPSS Symposium on Fusion Engineering, San Diego, 1991 (IEEE, New York, NY, 1992), vol. 2, p. 1199.

[14] T.S. Hahm, K.H. Burrell, Phys. Plasmas 2 (1995) 1648.

[15] K.H. Burrell, Phys. Plasmas 4 (1997) 1499.

[16] S. Coda, Ph.D. Thesis, Massachusetts Institute of Technology, 1997. Available from: http://theses.mit.edu:80/Dienst/UI/2.0/Describe /0018.mit.theses\%2f1997-322 [Accessed 18 July 2000].

[17] F. Zernike, Physica 1 (1934) 689.

[18] S. Coda, M. Porkolab, Rev. Sci. Instrum. 66 (1995) 454.

[19] S. Coda, M. Porkolab, in: Proc. 21th European Conference on Controlled Fusion and Plasma Physics, Montpellier, 1994 (European Physical Society, Petit-Lancy, Switzerland, 1994), vol. 1, p. 854 .

[20] K.H. Burrell et al., in: Proc. 15th International Conference on Plasma Physics and Controlled Nuclear Fusion Research, Sevilla, 1994 (International Atomic Energy Agency, Vienna, Austria, 1995), vol. 1, p. 221.

[21] T.S. Hahm, Phys. Plasmas 1 (1994) 2940.

[22] C.L. Rettig, Ph.D. Thesis, University of California, Los Angeles, 1993.

[23] This estimate is consistent with a majority of measurements in tokamak edges placing the value of $\mathscr{L}_{\theta} / \mathscr{L}_{r}$ between 1 and 3. See, e.g., Ch.P. Ritz et al., Phys. Fluids 27 (1984) 2956; S.J. Zweben, R.W. Gould, Nucl. Fusion 25 (1985) 171; R.J. Fonck et al., Plasma Phys. Control. Fusion 34 (1992) 1993.

[24] R.A. Moyer et al., Phys. Plasmas 2 (1995) 2397.

[25] P.H. Diamond et al., Phys. Rev. Lett. 72 (1994) 2565. 